\title{
Revisión sistemática sobre la prevalencia del síndrome de Burnout en el sector académico
}

\author{
Systematic review on the Burnout syndrome's prevalence in the academic \\ sector
}

\author{
Rosana Aurora Choy Vessoni ${ }^{1}$, Diego Eduardo Prieto Molinari ${ }^{2}$ \\ Universidad de Lima
}

Recibido: 03 - $11-21$

Aceptado: $30-11-21$

Publicado: $23-12-21$

\section{Resumen}

El síndrome de burnout tiene efectos negativos en los colaboradores de distintos sectores. No obstante, poco se afirma en relación a su prevalencia específica en el sector educativo. El presente estudio buscó definir la prevalencia del burnout en docentes y estudiantes según lo presentado por la literatura. Para ello, se realizó una revisión sistemática de investigaciones que reportan su prevalencia en el sector educativo, tanto para docentes como para estudiantes. Se obtuvo 1547 referencias únicas, de las cuales solo 59 reportaron adecuadamente información sobre prevalencia del síndrome de burnout en la población de interés. La proporción de niveles altos de cada dimensión mostró ser más elevada en estudiantes que en docentes. No obstante, la prevalencia del síndrome mostró resultados opuestos, siendo más frecuente en docentes $($ Mediana $=19.8 \%)$ que en estudiantes $($ Mediana $=14.4 \%)$. El estudio del burnout se ve afectado por algunas limitaciones; en particular, respecto al uso de instrumentos y puntos de corte. No obstante, la alta prevalencia de este problema en docentes y estudiantes es suficiente evidencia para profundizar el estudio de sus consecuencias en este sector específico.

Palabras clave: Burnout; educación; docentes; estudiantes; revisión sistemática.

\begin{abstract}
The burnout syndrome has negative consequences over employees in different areas. However, little is stated regarding its specific prevalence in the academic area. The present study seeks to define the prevalence of burnout in teachers and students as presented in the literature. A systematic review is conducted to research the prevalence of the burnout syndrome and its dimensions on the education sector, regarding both teachers and students. 1547 unique references were found, within which 59 reported information on the prevalence of the burnout syndrome in the population of interest. The proportion of high levels of each dimension showed to be higher in students than in teachers. However, the prevalence of the
\end{abstract}

\footnotetext{
1 Autor para correspondencia: rchoy@ulima.edu.pe ORCID: https://orcid.org/0000-0002-7807-9324

2 E-mail: dprieto@ulima.edu.pe ORCID: https://orcid.org/0000-0003-0470-5182
}

(C) Los autores. Este artículo es publicado por la Revista de Investigación en Psicología de la Facultad de Psicología, Universidad Nacional Mayor de San Marcos. Este es un artículo de acceso abierto, distribuido bajo los términos de la licencia Creative Commons Atribución 4.0 Internacional (CC BY 4.0) [https://creativecommons.org/licenses/by/4.0/deed.es] que permite el uso, distribución y reproducción en cualquier medio, siempre que la obra original sea debidamente citada de su fuente original. 
syndrome itself showed opposite results, being more frequent in teachers (Median $=19.8 \%$ ) than in students (Median $=14.4 \%$ ). Burnout research is affected by some limitations; particularly, regarding the use of psychometric instruments and cut-off points. Nevertheless, its high prevalence on both teachers and students are enough evidence to further research into its consequences in this specific sector.

Keywords: Burnout; education; teachers; students; systematic review. 
El síndrome de burnout ha sido definido por Maslach et al. (2001) como una inadecuada respuesta a la presencia crónica de estresores en el ambiente laboral con tres componentes principales: agotamiento emocional, despersonalización y ausencia de realización personal. Este síndrome parece ser más frecuente en contextos como el educativo o el de salud puesto que existe poca expectativa de control sobre los resultados y recompensas (Buunk \& Schaufeli, 1993), la necesidad de trabajo emocional (Jeung et al., 2018; Lee, 2019), y el riesgo de afectar las autoevaluaciones fundamentales de las personas (Leupold et al., 2020). Usualmente estudiado en el contexto organizacional, el agotamiento emocional es una respuesta ante la experiencia de sobrecarga laboral. La despersonalización o cinismo implica distanciamiento del cliente, paciente o estudiante para facilitar el afrontamiento de las demandas. Y la ausencia de realización personal implica la percepción de incompetencia, desempeño laboral disminuido y baja autoeficacia. Esta conceptualización, incluida en la Clasificación Internacional de Enfermedades [CIE] (WHO, 2019), descuida la ocurrencia del burnout en espacios distintos al de trabajo pero que también resultan de la presión excesiva (Rodriguez-Díaz, 2010) en sectores como el académico o el deportivo (Frajerman et al., 2019; Garcia-Jarillo et al., 2016; Robins et al., 2018).

Este síndrome parece ser un método no adaptativo de afrontar la exposición continua y prolongada al estrés en distintos contextos (Huyghebaert et al., 2018; Mandrick et al., 2016). El modelo de Demandas y Recursos explica su origen a través de dos procesos: a) desgaste del bienestar individual por las demandas laborales y b) promoción del bienestar individual por los recursos organizacionales (Adil \& Baig, 2018; Yin et al., 2018). Estas demandas y recursos se encuentran, principalmente, en el contexto institucional y tienen efectos sobre el servicio educativo ofrecido y la calidad de vida en el ambiente laboral (Dhir \& Shukla, 2019). Bajo este marco, se encuentran distintas variables relacionadas con el síndrome de burnout, como el estilo de liderazgo o el compromiso de los docentes (Buil et al., 2019; Caniëls et al., 2018); la rigidez en la cultura organizacional (Pedersen \& Minnotte, 2017); y la existencia de contextos laborales tóxicos (Rasool et al., 2019). El burnout fuera de ambientes laborales ha sido estudiado también bajo el mismo marco, mostrando resultados prometedores para su comprensión (Kim et al., 2015; Sorkkila et al., 2020; Wilkerson \& Bellini, 2006).

Por su parte, Salanova y colaboradores (2005) consideran necesario estudiar el rol de las diferencias individuales en el surgimiento de este síndrome. Es así que se ha estudiado el capital psicológico, como recurso individual y protector ante el síndrome de burnout (Demir, 2018). Además, se ha hallado que el Neuroticismo se relaciona positivamente con este síndrome; mientras que la Afabilidad parece cumplir un rol de factor protector (Conte et al., 2017; Khoeini \& Attar, 2015; Perera et al., 2018). Dicke y colaboradores (2018) plantean estudiar la percepción de exigencias como mediador de la relación entre la personalidad y el burnout. 
Otros autores enfatizan la necesidad de estudiar directamente la percepción de los recursos personales y organizacionales en docentes para prevenir el surgimiento del burnout (Bottiani et al., 2019; LePine et al., 2015). Otras variables que requieren mayor investigación incluyen la regulación emocional (Castillo-Gualda et al., 2019) y el Job Crafting (Rudolph et al., 2017; Teng \& Chen, 2019). Aunque, en general, las características individuales parecen ser más útiles para predecir el burnout (Jovanovic et al., 2020; Malinen \& Savolainen, 2016; Zysberg et al., 2017). No obstante, la relación entre recursos y surgimiento del burnout no es del todo clara; el potenciamiento inadecuado de algunos recursos puede resultar problemático (Huyghebaert et al., 2018; Upadyaya et al., 2016).

El impacto del contexto laboral y social sobre el individuo puede llegar a perjudicar su bienestar a nivel psicológico (Skaalvik \& Skaalvik, 2018) y fisiológico (Huyghebaert et al., 2018). Se relaciona con el surgimiento de síntomas de ansiedad, depresión e hipertensión; incluso, un aumento en el riesgo de desarrollar episodios de depresión mayor (Iancu et al., 2018; Skaalvik \& Skaalvik, 2018). Aumentan los problemas cardiovasculares y de tensión corporal, cefaleas y problemas del sueño (Paul III et al., 2017; Treviño et al., 2019). Existe, incluso, la preocupación de que este sea un predictor de suicidio (Frajerman et al., 2019). Además, se puede observar evitación de responsabilidades, desorganización y elevado consumo de cafeína, alcohol y tabaco (Raja et al., 2018). En el sector educativo, este malestar psicológico puede perjudicar el proceso de enseñanza-aprendizaje (Díaz et al., 2015; Reyes, 2016). En particular, se encuentra mayor ausentismo y menor productividad; menor satisfacción con el propio desempeño; mayor nivel de conflicto de rol; intención de renuncia y de deserción escolar; y, conductas de descortesía en el entorno (Adil \& Baig, 2018; Bask \& Salmela-Aro, 2013; Iancu et al., 2018; Rahim \& Cosby, 2016; Rasool et al., 2019; Zarei \& Dabaghi, 2019).

En cuanto a su prevalencia existen resultados variados dependiendo del estudio, aunque la mayoría de estos son reportados en el sector salud. Por ejemplo, en enfermería se ha encontrado alta prevalencia $(40.55 \%)$ de agotamiento emocional (Hakanen \& Bakker, 2017). En enfermería adjunta a la milicia se encontraron niveles variados $(10.5 \%$ - 21.1\%) de prevalencia de cada dimensión (Zarei \& Dabaghi, 2019). En obstetras, los resultados también son variados, aunque con mayor prevalencia $(18.8 \%$ - 31.3\%) en las dimensiones del burnout (Paredes, 2016). En doctores, hay menor prevalencia $(10.6 \%$ - 19.4\%) de niveles elevados de cada dimensión (Maticorena-Quevedo et al., 2016). En docentes, García-Carmona y sus colaboradores (2019) encuentran mayor prevalencia $(28.1 \%$ - $40.3 \%)$ de altos niveles de cada dimensión. Además, señalan que la prevalencia del burnout en este sector debería ser más estudiado y reportado (García-Carmona et al., 2019). Maticorena-Quevedo y colaboradores (2016) resaltan la importancia de reportar también los puntos de corte utilizados para determinar la prevalencia. Hakanen \& Bakker (2017) considera que, tras 40 años de investigación, todavía es necesario 
el estudio del síndrome de burnout. Esto da lugar a que los autores se pregunten cuál es la prevalencia del síndrome de burnout y sus dimensiones en docentes y estudiantes de distintos niveles educativos.

\section{MÉTODO}

\section{Procedimiento}

Con el objetivo de brindar una revisión del estado de investigación reproducible y con el menor nivel posible de sesgo, se decide hacer uso de algunos criterios y estándares sugeridos por Perestelo-Pérez (2013). En ese sentido, se genera la primera pre-selección de documentos a través del uso de buscadores bases de datos electrónicas. La fórmula booleana usada en los campos de búsqueda de título o palabras clave fue [" "teacher burnout" OR "student burnout" OR "academic burnout" OR "burnout") AND ("prevalence" OR "frequency" OR "incidence")]. Se utilizaron las bases electrónicas APA PsycNET y Psicodoc, relacionadas a la psicología; y, Ebsco y SciELO como bases multidisciplinarias en tanto que este tema de investigación puede ser enmarcado en la educación y en la gestión del recurso humano. Como filtro se consideró pertinente revisar solo las publicaciones realizadas desde el 2016 hasta abril del 2021, ya sea que estas fueran de acceso abierto o de acceso pago. La información recopilada se organizó en una base de datos electrónica. En esta se sistematizó información ligada a la autoría y año de publicación; información sobre la muestra, tamaño, edad, tipo de población y nivel educativo; información ligada al método, el instrumento utilizado, la realización de modificaciones, y el reporte de análisis psicométrico. Finalmente, con respecto al síndrome de burnout, se recolectó información ligada a la prevalencia de niveles altos de burnout y sus dimensiones.

\section{Criterios de inclusión y exclusión}

Con el objetivo de delimitar la búsqueda de artículos y asegurar la calidad y pertinencia de los estudios encontrados, se plantean algunos criterios de inclusión y exclusión. En primer lugar, los participantes de las investigaciones deben ser estudiantes o docentes, procedentes de población no clínica. Además, se incluyó solo investigaciones cuantitativas, publicadas como artículos científicos, reportes cortos o tesis doctorales o de maestría. Por otro lado, se descartó investigaciones que no permitan identificar la población evaluada. Además, se descartó investigaciones que no reporten información de los instrumentos utilizados; e investigaciones secundarias, como revisiones o meta-análisis.

\section{Consideraciones éticas}

Tratándose de una revisión sistemática de la bibliografía se hizo uso de bases de datos electrónicas y filtros. Por ello se plantean criterios de inclusión y exclusión, así como rigurosidad y orden en el proceso de filtro y selección de las 
investigaciones para brindar un resultado sin sesgos. Además, se citaron debidamente todos los trabajos utilizados para el desarrollo del presente estudio y la información extraída de ellos no ha sido alterada a conveniencia del estudio actual.

\section{RESULTADOS}

La muestra de artículos, descrita en la Figura 1, procede de distintas bases electrónicas: APA PsycNet (35), Psicodoc (31), Ebsco (1787) y Scielo (151). Alrededor del $90 \%$ de las investigaciones únicas obtenidas se relacionaban al burnout en personal del sector salud, atletas, pacientes, guías turísticos, personal en centros penitenciarios, entre otros. Solo el 3.81\% de los artículos reportan datos relacionados al burnout en el ámbito académico.

\section{Figura 1}

Proceso de búsqueda y selección de investigaciones

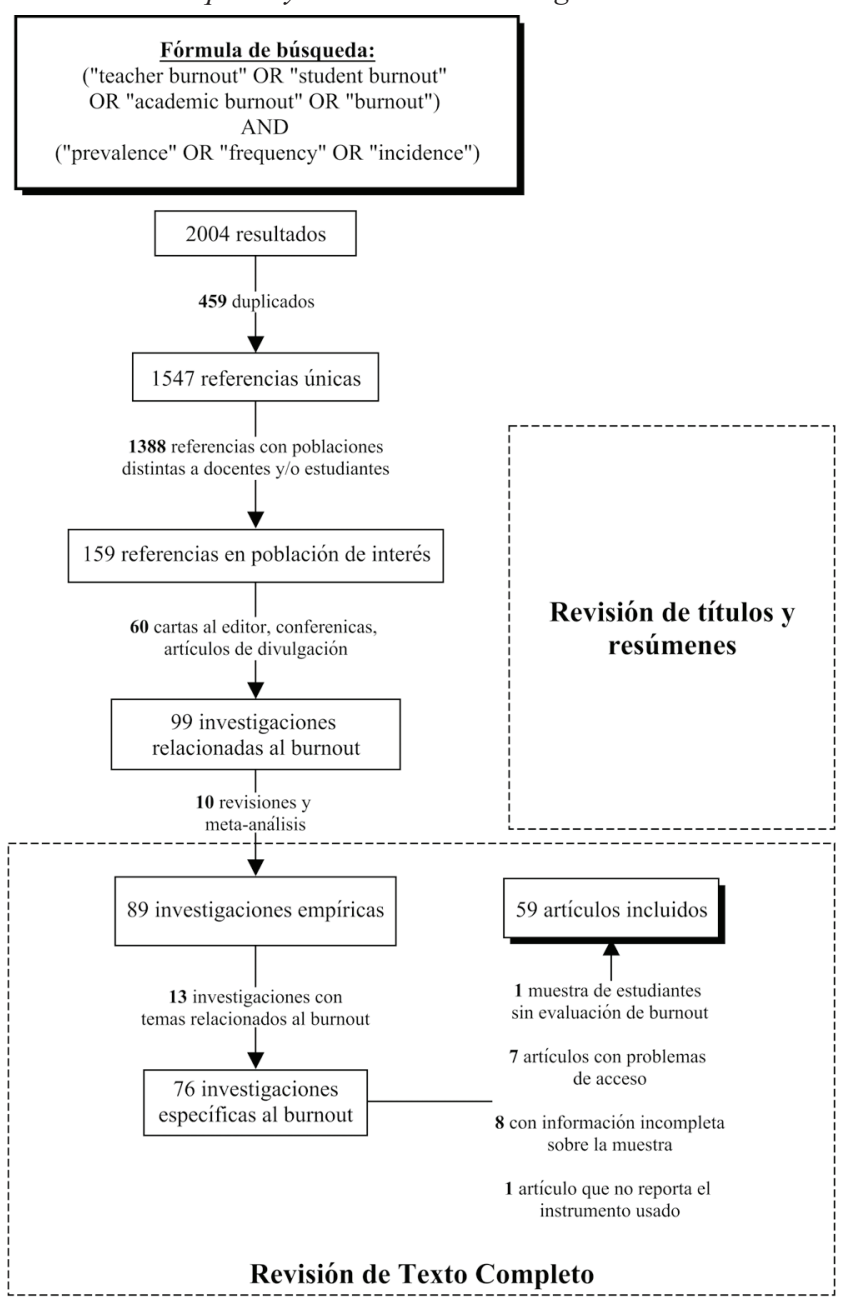


En los 59 artículos revisados se afirma la prevalencia de niveles altos de burnout en muestras de docentes yendo desde $0 \%$ a $53.8 \%$ (Mediana $=19.8 \%$ ); mientras que, en muestras de alumnos, estos porcentajes van desde $0 \%$ hasta $67.9 \%$ $($ Mediana $=14.4 \%)$. Se enfatiza que solo el $71.2 \%$ de las investigaciones reporta porcentajes de prevalencia; y, de estas, menos de la mitad (46.55\%) reporta la estrategia utilizada para definirla. Alrededor del $20 \%$ de los que sí la reportan, hacen uso de puntajes arbitrarios o predefinidos para definir la prevalencia de niveles "Alto", "Moderado" y "Bajo"; 13.73\% hacen uso de baremos incluidos en los manuales de los instrumentos; y solo el 1.96\% hace uso de puntos de cortes obtenidos con análisis de curva ROC. En docentes se reporta la prevalencia de agotamiento emocional en proporciones desde el $0 \%$ hasta el 55.5\% (Mediana $=23.5 \%$ ); niveles altos de despersonalización desde el $0 \%$ hasta el $67.8 \%$ (Mediana $=16.6 \%$ ); y niveles bajos de realización personal desde el $0 \%$ hasta el 65.7\% (Mediana $=14.2 \%)$. En estudiantes, la prevalencia de niveles altos de agotamiento emocional va desde $0 \%$ hasta $80.3 \%$ (Mediana $=35.6 \%$ ), niveles altos de despersonalización o cinismo desde $0 \%$ hasta $58 \%$ (Mediana $=35.75 \%$ ); y niveles bajos de realización personal o entusiasmo desde $0 \%$ y hasta $58.9 \%$ (Mediana $=33.7 \%$ ). Es importante también mencionar que alrededor del $30 \%$ de las investigaciones que proveen datos ligados a los niveles de burnout solo reportan puntajes directos obtenidos con los cuestionarios. Finalmente, se encuentra que alrededor del $20 \%$ de las investigaciones realiza modificaciones a los instrumentos o hace uso de técnicas cuyos puntajes no han mostrado previamente evidencias de validez en muestras similares. De hecho, solo el $35.48 \%$ de las investigaciones reportó haber realizado algún análisis para obtener evidencias de validez de los puntajes; de hecho, solo la mitad de aquellos que usaron instrumentos modificados o construidos para la investigación reportan haberlos realizado (ver Tabla 1 y 2).

Tabla 1

Prevalencia de Sindrome de burnout en estudiantes

\begin{tabular}{llllcccc}
\hline Investigación & $\mathbf{N}$ & Nivel & Instrumento & \%SB & \%AE & \%D & \%AR \\
\hline Aboalshamat et al. (2017) & 645 & Universitario & CBI & 67.9 & - & - & - \\
Uribe \& Illesca (2017) & 244 & Universitario & EUBE & 0 & 0 & 0 & 0 \\
Sorrel et al. (2020) & 234 & Posgrado & MBI & - & 80.3 & 58 & 58.9 \\
Mejia et al. (2016) & 557 & Universitario & MBI & 8 & - & - & - \\
Vilchez-Cornejo et al. (2016) & 583 & Universitario & MBI & 24.1 & 16.5 & 47.7 & 35.3 \\
Merchán-Galvis et al. (2018) & 161 & Universitario & MBI & 4.3 & 37.9 & 23 & 24.8 \\
Almeida et al. (2016) & 376 & Universitario & MBI-HSS & 14.9 & - & - & - \\
Padovani et al. (2016) & 468 & Universitario & MBI-SS & 5 & - & - & - \\
Amor et al. (2020) & 149 & & & 33.6 & 65.1 & 37.6 & 34.2 \\
Cazolari et al. (2020) & 224 & Universitario & MBI-SS & 38 & 68.3 & 45.1 & 40.6 \\
González-Escobar et al. (2020) & 120 & Universitario & MBI-SS & 12 & - & - & - \\
Magri et al. (2019) & 57 & Universitario & MBI-SS & 8.7 & 33.3 & 33.3 & 33.3 \\
\hline
\end{tabular}


Tabla 1: Continuación...

\begin{tabular}{llllcccc}
\hline Investigación & $\mathbf{N}$ & Nivel & Instrumento & \%SB & \%AE & \%D & \%AR \\
\hline Puertas-Neyra et al. (2020) & 269 & Universitario & MBI-SS & 32.3 & 58.7 & 14.1 & 46.1 \\
Al-Alawi et al. (2019) & 662 & Universitario & MBI-SS & 7.4 & 30.1 & 33.9 & 32.1 \\
Estrada-Araoz et al. (2021) & 202 & Universitario & MBI-SS & 25.2 & 16.4 & 26.7 & 33.7 \\
Wickramasinghe et al. (2018) & 796 & Secundaria & MBI-SS & 28.8 & - & - & - \\
Ali et al. (2018) & 373 & Universitario & OLBI & 61.1 & 58.7 & 56.3 & - \\
Salmela-Aro et al. (2016) & 255 & \multirow{2}{*}{ Secundaria } & SBI & 7.8 & - & - & - \\
& 188 & & & 14.4 & - & - & - \\
\hline
\end{tabular}

Nota EUBE = Escala Unidimensional del Burnout Estudiantil; OLBI = Oldenburg Burnout Inventory; CBI = Copenhagen Burnout Inventory; MBI-HSS = Maslach Burnout Inventory - Human Services Survey; MBI-SS = Maslach Burnout Inventory - Student Survey; SBI = School Burnout Inventory

\section{Tabla 2}

Prevalencia de Síndrome de burnout en docentes

\begin{tabular}{|c|c|c|c|c|c|c|c|}
\hline Investigación & $\mathbf{N}$ & Nivel & Instrumento & $\% \mathrm{SB}$ & $\% \mathbf{A E}$ & $\% \mathrm{D}$ & $\% A R$ \\
\hline Gallardo-López et al. (2019) & 1890 & Básica & CBP-R & 11.6 & 25.9 & 16.9 & 0 \\
\hline Unda-Rojas et al. (2020) & 395 & Universitario & CESQT & 2 & 20.7 & 10 & 11.7 \\
\hline Reyes-Oyola \& Palomino-Devia (2019) & 111 & Básica & CESQT-PE & 19.8 & 34.2 & 24.3 & 1.8 \\
\hline Villamar-Sánchez et al. (2019) & 247 & Universitario & CESQT-PE & 30 & 10.1 & 7.3 & 0.4 \\
\hline S. Silva \& Oliveira (2019) & 179 & Universitario & ECB & 20.8 & 29.5 & 34.7 & 34.7 \\
\hline N. Silva et al. (2018) & 100 & Básica & ISB & 29 & 37 & 22 & 11 \\
\hline Akman (2016) & 423 & - & JBS & 0 & 0 & 0 & 0 \\
\hline Capelo (2017) & 119 & Pre-Escolar & MBI & 0 & 0 & 0 & 0 \\
\hline Zuniga-Jara \& Pizarro-Leon (2018) & 49 & Básica & MBI & - & 55.1 & 16.3 & 38.8 \\
\hline Akkaya \& Serin (2020) & 393 & Universitario & MBI & - & 0 & 0 & 0 \\
\hline Capone \& Petrillo (2020) & 280 & Secundaria & MBI & - & 22.1 & 9.5 & - \\
\hline Malander (2019) & 123 & Secundaria & MBI & 2.44 & 17.1 & 5.7 & 31.7 \\
\hline Ramos-Argilagos et al. (2020) & 70 & Universitario & MBI & 53.8 & 55.7 & 60 & 65.7 \\
\hline Sánchez-Narváez \& Velazco-Orozco (2017) & 459 & Básica & MBI-ED & 25.9 & 24.8 & 38.3 & 13.7 \\
\hline Quintero-Idárraga \& Hernández-Calle (2020) & 194 & Básica & MBI-ES & - & 29.9 & 17.6 & 17.6 \\
\hline Carvalho de Sousa et al. (2020) & 103 & - & MBI-ES & 41 & 43.7 & 22.3 & 53.4 \\
\hline Jeunon et al. (2017) & 309 & Universitario & MBI-ES & - & 2 & 3 & 16.5 \\
\hline Shaheen \& Mahmood (2016) & 416 & Básica & MBI-ES & - & 14.4 & 14.9 & 72.6 \\
\hline Acosta-Fernández et al. (2019) & 68 & Universitario & MBI-HSS & 11.8 & 0 & 0 & 14.7 \\
\hline Kebde \& Gedfie (2018) & 298 & Universitario & MBI-HSS & - & 55.5 & 67.8 & 65.1 \\
\hline Carlotto \& Câmara (2019) & 679 & Primaria & SBI-ED & 7.5 & 15.3 & 20.9 & 10.9 \\
\hline
\end{tabular}

Nota. ECB = Escala de Caracterização do Burnout; CBP-R = Cuestionario de Burnout del Profesorado; $\mathrm{JBS}=$ Job Burnout Scale; MBI-HSS = Maslach Burnout Inventory - Human Services Survey; MBI-ES = Maslach Burnout Inventory - Educators Survey; MBI-ES = Maslach Burnout Inventory; CESQT-PE = Cuestionario para la evaluación del síndrome de quemarse con el trabajo (Profesionales de Educación); CESQT = Cuestionario para la evaluación del síndrome de quemarse con el trabajo; SBI-ED = Spanish Burnout Inventory for education professionals. 


\section{DISCUSIÓN}

El objetivo del presente estudio es analizar la prevalencia del síndrome de burnout y sus dimensiones en el sector académico según la literatura de los últimos cinco años; específicamente en docentes y estudiantes. Al respecto, es un hallazgo de interés que la prevalencia reportada de las dimensiones de burnout y del síndrome es bastante variada en ambas poblaciones. En base a la mediana de prevalencia, se puede observar que la población docente muestra mayor prevalencia del síndrome que los estudiantes. No obstante, la prevalencia de cada dimensión parece ser más elevada para estudiantes que docentes. Este resultado contraintuitivo parece resaltar la necesidad de revisar las estrategias utilizadas para determinar su prevalencia. Por otro lado, se puede encontrar que los niveles reportados en ambas poblaciones son semejantes, o incluso mayores en el caso de los estudiantes, a los reportados en el sector salud (Maticorena-Quevedo et al., 2016; Paredes, 2016; Zarei \& Dabaghi, 2019). Si bien esta clase de comparaciones no resulta adecuada puesto que no se está tomando en cuenta la comparabilidad de las escalas ni el supuesto de invarianza poblacional, este ejercicio revela que la prevalencia de este síndrome requiere de atención en investigaciones empíricas y secundarias como metaanálisis.

Si bien estos resultados coinciden con lo planteado por otros autores; aún es necesario estudiar el síndrome de burnout y su prevalencia en docentes y estudiantes (García-Carmona et al., 2019; Maticorena-Quevedo et al., 2016). Existen limitaciones para la revisión de evidencia. De modo particular, se señala que solo una pequeña fracción de las investigaciones halladas en los últimos cinco años se enfocan en este sector; e incluso entre ellas, algunas solo hacen uso de la variable para análisis correlacionales y construcción de teoría sin reportar información ligada a su prevalencia. La inclusión de este síndrome en la CIE (WHO, 2019), aumenta la importancia de generar herramientas adecuadas para su diagnóstico. Hasta el momento, esta variable ha sido estudiada como una variable continua o enmarcada en niveles de prevalencia con estrategias variadas e instrumentos igual de diversos. La evidencia hallada despierta ciertas preocupaciones con respecto a la validez de sus puntajes y las inferencias realizadas a partir de ellos. En primer lugar, se puede observar que gran cantidad de investigaciones hace uso de herramientas "previamente adaptadas" y omite la revisión de las propiedades psicométricas de los puntajes en la muestra. Si bien la "validación" puede no ser la finalidad de un artículo, es importante tomar en cuenta que las propiedades psicométricas no competen a los instrumentos y que generalizar las evidencias de validez obtenidas en una muestra hacia otra puede ser inadecuado. Más allá de eso, se encuentra que algunas investigaciones realizan modificaciones a los instrumentos y no reportan realizar análisis psicométricos. Finalmente, resulta preocupante observar que algunas investigaciones reportan la prevalencia en base a puntajes arbitrarios o baremos obtenidos durante la construcción original del cuestionario sin considerar 
que los puntajes pueden funcionar de modo diferente en muestras diferentes. No solo eso, sino que algunas investigaciones omiten reportar la estrategia usada para determinar el nivel de prevalencia del síndrome y sus dimensiones, lo cual genera problemas ligados a la replicabilidad y transparencia de la investigación en el campo como ha sido señalado ya por Maticorena-Quevedo y colaboradores (2016).

Ignorando los aspectos metodológicos y las limitaciones de la investigación, los resultados hallados permiten resaltar la urgencia de trabajar en la prevención y tratamiento del síndrome de burnout en el sector académico. Y, más aún, permiten resaltar la necesidad de atender a este síndrome en áreas de la vida ajenas a lo laboral. El modelo de Recursos y Demandas Laborales ha permitido generar estrategias para prevenir y reducir la incidencia de este síndrome (Adil \& Baig, 2018). En particular, Vîrgă et al. (2020) explican que se puede potenciar los recursos personales en la organización; no obstante, otros autores plantean que se debe generar una disminución de las demandas y cambios estructurales sobre el sistema de producción, no exclusivamente laboral, para lograrlo. El reporte de la prevalencia, así como la obtención de evidencias de validez de los puntajes y de sus puntos de corte son aspectos de suma importancia para el correcto diagnóstico e intervención sobre esta afección (Swensen \& Shanafelt, 2017; Van den Broeck et al., 2017).

\section{CONCLUSIONES}

En base a los resultados obtenidos, y el objetivo de conocer la prevalencia del síndrome de burnout en el sector académico, se puede concluir que este síndrome afecta alrededor del 19.8\% de la población docente; mientras que al 14.4\% de los estudiantes. A pesar de ello, se encuentra mayor prevalencia de cada subdimensión, siendo así que el Agotamiento emocional está presente en el 35.6\% de los estudiantes y el $23.5 \%$ de los docentes. La despersonalización prevalece en el $35.75 \%$ de los estudiantes y en el $16.6 \%$ de los docentes. Finalmente, la Ausencia de Realización se encuentra en el $33.7 \%$ de los estudiantes; mientras que en el $14.2 \%$ de los docentes.

\section{Rol de los autores:}

RACV: Antecedentes; método; búsqueda, sistematización y revisión de artículos; discusión y conclusiones

DEPM: Antecedentes; método; búsqueda, sistematización y revisión de artículos; discusión y conclusiones

\section{Conflicto de intereses}

Los autores del presente artículo informan que no existe ningún tipo de conflictos de interés. 


\section{REFERENCIAS}

*Aboalshamat, K., Alzahrani, M., Rabie, N., Alharbi, R., Joudah, R., Khulaysi, S., \& Alansari, W. (2017). The Relationship between Burnout and Perfectionism in Medical and Dental Students in Saudi Arabia. Journal of Dental Specialities, 5(2), 122127.

*Acosta-Fernández, M., Parra-Osorio, L., Burbano-Molina, C., Aguilera-Velasco, M. de los Á., \& Pozos-Radillo, B. E. (2019). Estrés laboral, burnout, salud mental y su relación con violencia psicológica en docentes universitarios. Revista Salud Uninorte, 35(3), 328-342.

*Akkaya, A., \& Serin, H. (2020). The Investigation of the Relationship Between Academics' Person-Organization Fit and Burnout Levels. International Journal of Progressive Education, 16(4), 27-44.

*Akman, Y. (2016). The Relationship between Destructive Leadership and Job Burnout: A Research on Teachers. Yikici Liderlik Ile Mesleki Tükenmişlik Arasindaki İlişki: Öğretmenler Üzerine Bir Araştirma., 12(3), 627-653.

*Al-Alawi, M., Al-Sinawi, H., Al-Qubtan, A., Al-Lawati, J., Al-Habsi, A., Al-Shuraiqi, M., Al-Adawi, S., \& Panchatcharam, S. M. (2019). Prevalence and Determinants of Burnout Syndrome and Depression among Medical Students at Sultan Qaboos University: A Cross-Sectional Analytical Study from Oman. Archives of Environmental \& Occupational Health, 74(3), 130-139.

*Ali, M., Liaqat, A., Sethi, M. R., \& Irfan, M. (2018). Effect of Social Support on Burnout in Medical Students. JPMI: Journal of Postgraduate Medical Institute, 32(3), 266270.

*Almeida, G. de C., Souza, H. R. de, Almeida, P. C. de, Almeida, B. de C., \& Almeida, G. H. (2016). The Prevalence of Burnout Syndrome in Medical Students. Archives of Clinical Psychiatry (São Paulo), 43(1), 6-10. https://doi.org/10.1590/010160830000000072

*Amor, E. M., Baños, J. E., \& Sentí, M. (2020). Prevalencia del síndrome de burnout entre los estudiantes de medicina y su relación con variables demográficas, personales y académicas. FEM: Revista de la Fundación Educación Médica, 23(1), 25-33.

*Bradley, C., Cordaro, D. T., Zhu, F., Vildostegui, M., Han, R. J., Brackett, M., \& Jones, J. (2018). Supporting Improvements in Classroom Climate for Students and Teachers with the Four Pillars of Wellbeing Curriculum. Translational Issues in Psychological Science, 4(3), 245-264. https://doi.org/10.1037/tps0000162

*Capelo, M. R. T. F. (2017). Vulnerabilidade ao estresse, coping e burnout em educadoras de infância portuguesas. Educar em Revista, 64, 155-169. https://doi.org/10.1590/0104$\underline{4060.49793}$

*Capone, V., \& Petrillo, G. (2020). Mental Health in Teachers: Relationships with Job Satisfaction, Efficacy Beliefs, Burnout and Depression. Current Psychology, 39(5), 1757-1766. 
*Carlotto, M. S., \& Câmara, S. G. (2019). Prevalence and Predictors of Burnout Syndrome among Public Elementary School Teachers. Análise Psicológica, 37(2), 135-146. https://doi.org/10.14417/ap.1471

*Carson, R., Baumgartner, J., Ota, C., Kuhn, A., \& Durr, A. (2017). An Ecological Momentary Assessment of Burnout, Rejuvenation Strategies, Job Satisfaction, and Quitting Intentions in Childcare Teachers. Early Childhood Education Journal, 45(6), 801-808.

*Carvalho de Sousa, J., Barra de Oliveira, A. M., Medeiros da Silva, P. M., \& Pinto Brito, L. M. (2020). Burnout in Teaching Activity: Evidence of a Study Involving Higher Education Institutions. Burnout na atividade docente: evidências de um estudo envolvendo instituições de ensino superior., 13(3), 554-565.

*Cazolari, P. G., Cavalcante, M. de S., Demarzo, M. M. P., Cohrs, F. M., Sanudo, A., \& Schveitzer, M. C. (2020). Burnout and Well-Being Levels of Medical Students: A Cross-Sectional Study. Revista Brasileira de Educação Médica, 44(4). https://doi. org/10.1590/1981-5271v44.4-20190138.ing

*Chust-Hernández, P., Castellano-Rioja, E., Fernández-García, D., \& Chust-Torrent, J. I. (2019). Ansiedad Ante Los Exámenes En Estudiantes de Enfermería: Factores de Riesgo Emocionales y de Sueño. Ansiedad y Estrés, 25(2), 125-131. https://doi. org/10.1016/j.anyes.2019.05.001

*Cornér, S., Löfström, E., \& Pyhältö, K. (2017). The Relationships between Doctoral Students' Perceptions of Supervision and Burnout. International Journal of Doctoral Studies, 12, 91-106.

*Demirel, E. E., \& Erdirençelebi, M. (2019). The Relationship of Burnout with Workaholism Mediated by Work-Family Life Conflict: A Study on Female Academicians. Tükenmişliğin iş-aile yaşam çatışması aracılığıyla işkoliklikle ilişkisi: Kadın akademisyenler üzerine bir araştırma., 15(4), 1300-1316.

*Estrada-Araoz, E. G., Gallegos-Ramos, N. A., \& Mamani-Uchasara, H. J. (2021). Burnout Académico En Estudiantes Universitarios Peruanos. (Spanish). Academic burnout in Peruvian university students. (English), 11(2), 48-62.

*Gallardo-López, J. A., López-Noguero, F., \& Gallardo-Vázquez, P. (2019). Análisis del síndrome de burnout en docentes de educación infantil, primaria y secundaria para su prevención y tratamiento. Revista Electrónica Educare, 23(2), 324-343. https://doi. org/10.15359/ree.23-2.17

*González-Escobar, J. M., Ramos-Franco Netto, R. O., de Almeida Rodrigues-Franco Netto, J., Flores, B. H., Borges Andreo, S., \& Coronel-de Bobadilla, B. (2020). Prevalencia del Síndrome de Burnout en Estudiantes de Medicina. Revista del Instituto de Medicina Tropical, 15(2), 13-18. https://doi.org/10.18004/imt/2020.15.2.13

*Jeunon, E. E., dos Santos Correa, L. Á., Duarte, L. da C., \& Rodrigues Guimarães, E. H. (2017). Burnout Syndrome: A Study with Professors at a Public University in Maranhão. Síndrome de Burnout: un estudio con docentes en una universidad pública de Maranhão., 17(3), 252-268. 
*Kebde, A., \& Gedfie, M. (2018). Burnout Experience among Public Universities of Amhara Regional State, Ethiopia. Indian Journal of Health \& Wellbeing, 9(8/9), 929-940.

*Klussman, K., Lindeman, M. I. H., Nichols, A. L., \& Langer, J. (2020). Fostering Stress Resilience Among Business Students: The Role of Stress Mindset and Self-Connection. Psychological Reports, 1.

*Magri, L. V., Melchor, M. de O., Jarina, L., Simonaggio, F. F., \& Bataglion, C. (2019). Temporomandibular Disorders and Burnout Syndrome in Students of the Eighth Period of Dentistry Course. RGO - Revista Gaúcha de dontología, 67. https://doi. org/10.1590/1981-863720190005520180011

*Malander, N. (2019). Prevalencia de Burnout En Docentes de Nivel Secundario. Prevalence of burnout in highschool teachers., 15(29), 84-99.

*May, R. W., Seibert, G. S., Sanchez-Gonzalez, M. A., \& Fincham, F. D. (2018). School Burnout and Heart Rate Variability: Risk of Cardiovascular Disease and Hypertension in Young Adult Females. Stress: The International Journal on the Biology of Stress, 21(3), 211-216.

*Maymon, R., Hall, N. C., \& Harley, J. M. (2019). Supporting First-Year Students During the Transition to Higher Education: The Importance of Quality and Source of Received Support for Student Well-Being. Student Success, 10(3), 64-75.

*Mejia, C. R., Valladares-Garrido, M. J., Talledo-Ulfe, L., Sánchez-Arteaga, K., Rojas, C., Arimuya, J. J., Cruz, B., \& Paredes Vilca, Z. (2016). Síndrome de Burnout y factores asociados en estudiantes de medicina: Estudio multicéntrico en siete facultades de medicina peruanas. Revista chilena de neuro-psiquiatría, 54(3), 207-214.

*Merchán-Galvis, Á. M., Albino Matiz, A. Y., Bolaños-López, J. E., Millán, N., \& AriasPinzón, A. A. (2018). Síndrome de Burnout y Factores Asociados En Estudiantes de Medicina. Burnout Syndrome and associated factors in Medical students., 32(3), 172-180.

*Meredith, C., Schaufeli, W., Struyve, C., Vandecandelaere, M., Gielen, S., \& Kyndt, E. (2020). 'Burnout Contagion' among Teachers: A Social Network Approach. Journal of Occupational \& Organizational Psychology, 93(2), 328-352.

*Molina-Torres, G., Roman, P., Butilca, A., Sánchez-Labraca, N., Cardona, D., \& Gonzalez-Sanchez, M. (2020). Relationship between Temporomandibular Disorders and Psychological and Sleep Aspects in University Teaching Staff: A Regression Model. Journal of Clinical Medicine, 9(12), 3960.

*Muñoz-Campos, F., Medina-Moreno, A., Carrasco-Mardones, D., Pérez-Villalobos, C., \& Ortiz-Moreira, L. (2016). Burnout En Estudiantes de Odontología y Su Relación Con Sus Características Sociodemográficas y Antecedentes Académicos. (Spanish). Burnout in Dentistry students and its relationship with their social-demographic characteristics and academic antecedents. (English), 30(2), 113-124.

*Padovani, R. da C., Neufeld, C. B., Maltoni, J., Barbosa, L. N. F., Souza, W. F. de, Cavalcanti, H. A. F., \& Lameu, J. do N. (2016). Vulnerabilidade e Bem-Estar Psicológicos Do Estudante Universitário. Revista Brasileira de Terapias Cognitivas, 10(1), 2-10. https://doi.org/10.5935/1808-5687.20140002 
*Puertas-Neyra, K., Mendoza T, G., Cáceres L, S., \& Falcón P, N. (2020). Síndrome de Burnout en estudiantes de Medicina Veterinaria. Revista de Investigaciones Veterinarias del Perú, 31(2). https://doi.org/10.15381/rivep.v31i2.17836

*Quintero-Idárraga, S., \& Hernández-Calle, J. A. (2020). Síntomas de Depresión Asociados al Síndrome de Burnout y a Condiciones Socio-Laborales de Docentes de Colegios Públicos de Envigado-Colombia. Psicología desde el Caribe [edición electrónica], 38(1).

*Ramos-Argilagos, M., Alfonso-González, I., Peñafiel-Jaramillo, K., Macias-Silva, E. C., \& Labrada-González, E. (2020). Síndrome de Burnout En Docentes de Dos Universidades de Ecuador. (Spanish). Burnout syndrome in teachers of two universities in Ecuador. (English), 7(2), 1-16.

*Reyes-Oyola, F. A., \& Palomino-Devia, C. (2019). Síndrome de desgaste profesional, índice de masa corporal y otros factores asociados con la labor de profesores de educación física de Ibagué, Colombia. Biomédica, 39(3), 537-546. https://doi.org/10.7705/ biomedica.4282

*Salgado, S., \& Au-Yong-Oliveira, M. (2021). Student Burnout: A Case Study about a Portuguese Public University. Education Sciences, 11(1), 31-31.

*Salmela-Aro, K., Moeller, J., Schneider, B., Spicer, J., \& Lavonen, J. (2016). Integrating the Light and Dark Sides of Student Engagement Using Person-Oriented and Situation-Specific Approaches. Learning \& Instruction, 43, 61-70.

*Sánchez-Narváez, F., \& Velazco-Orozco, J. J. (2017). Comorbilidad entre síndrome de Burnout, depresión y ansiedad en una muestra de profesores de Educación Básica del Estado de México. Papeles de población, 23(94), 261-286. https://doi.org/10.22185/2 4487147.2017.94.038

*Santos, A., \& Tin, J. J. (2018). The Nature, Extent and Impact of Educator Targeted Bullying on School Teachers in West Malaysia. British Journal of Guidance \& Counselling, 46(5), 543-556.

*Sert, M. (2018). An Analysis on the Secondary School Students Burnout in Terms of Academic Self-Efficacy, Parental Monitoring, Social Support, and Leisure Time Activities. International Journal of Eurasia Social Sciences / Uluslararasi Avrasya Sosyal Bilimler Dergisi, 9(34), 2313-2341.

*Seth, A. (2016). Study of Mental Health and Burnout in Relation to Teacher Effectiveness among Secondary School Teachers. Indian Journal of Health \& Wellbeing, 7(7), 769-773.

*Shaheen, F., \& Mahmood, N. (2016). Demographic Variables as Determinants of Emotional Burnout among Public School Teachers. Journal of Research \& Reflections in Education (JRRE), 10(1), 37-50.

*Silva, N., Bolsoni-Silva, A., \& Loureiro, S. R. (2018). Burnout e Depressão Em Professores Do Ensino Fundamental: Um Estudo Correlacional. Revista Brasileira de Educação, 23. https://doi.org/10.1590/s1413-24782018230048 
*Silva, S., \& Oliveira, Á. (2019). Burnout Em Professores Universitários Do Ensino Particular. Psicologia Escolar e Educacional, 23, 1-10. https://doi.org/10.1590/2175$\underline{35392019017785}$

*Síncer, S., \& Atanur-Baskan, G. (2017). Analyzing the Relationship Between Culture of Fear and Burnout According to the Perceptions of Academics. Ögretim Elemanlarl Algılarına Göre Korku Kültürü ile Tükenmişlik Arasındaki İlişkinin Incelenmesi., $7(3), 462-472$.

*Sorrel, M. A., Martínez-Huertas, J. Á., \& Arconada, M. (2020). It Must Have Been Burnout: Prevalence and Related Factors among Spanish PhD Students. The Spanish Journal of Psychology, 23, 1-13. https://doi.org/10.1017/SJP.2020.31

*Unda-Rojas, S., Hernández Toledano, R. A., García Arreola, O., \& Esquivel Lozada, C. (2020). Factores de Riesgo Psicosocial Predictores Del Síndrome de Quemarse Por El Trabajo (SQT) (Burnout) En Docentes de Bachillerato. Informació Psicolòica, 119, 91-107. https://doi.org/10.14635/IPSIC.2020.119.1

*Uribe, M. Á., \& Illesca, M. (2017). Burnout en estudiantes de enfermería de una universidad privada. Investigación en educación médica, 6(24), 234-241. https://doi. org/10.1016/j.riem.2016.11.005

*Vilchez-Cornejo, J., Huamán-Gutiérrez, R., Arce-Villalobos, L., Morán-Mariños, C., Mihashiro-Maguiña, K., Melo-Mallma, N., Eyzaguirre-Villagarcia, J., \& Rojas-Va1le, C. (2016). Síndrome de burnout en estudiantes de medicina: frecuencia, características y factores asociados. Acta Médica Peruana, 33(4), 282-288.

*Villamar-Sánchez, D., Juárez-García, A., González-Corzo, I. G., \& Osnaya-Moreno, M. (2019). Factores psicosociales y síndrome de Burnout en académicos de una universidad pública de México. Propósitos y Representaciones, 7(3), 111-126. https://doi. org/10.20511/pyr2019.v7n3.360

*Wang, Q., Hu, W., Ouyang, X., Chen, H., Qi, Y., \& Jiang, Y. (2020). The Relationship between Negative School Gossip and Suicide Intention in Chinese Junior High School Students: The Mediating Effect of Academic Burnout and Gender Difference. Children \& Youth Services Review, 117.

*Wickramasinghe, N. D., Dissanayake, D. S., \& Abeywardena, G. S. (2018). Prevalence and Correlates of Burnout among Collegiate Cycle Students in Sri Lanka: A SchoolBased Cross-Sectional Study. Child \& Adolescent Psychiatry \& Mental Health, 12(1), N.PAG-N.PAG.

*Williams, C. J., Dziurawiec, S., \& Heritage, B. (2018). More Pain than Gain: EffortReward Imbalance, Burnout, and Withdrawal Intentions within a University Student Population. Journal of Educational Psychology, 110(3), 378-394. https://doi. org/10.1037/edu0000212

*Zhang, Q., Yin, J., Chen, H., Zhang, Q., \& Wu, W. (2020). Emotional Labor Among Early Childhood Teachers: Frequency, Antecedents, and Consequences. Journal of Research in Childhood Education, 34(2), 288-305. 
*Zuniga-Jara, S., \& Pizarro-Leon, V. (2018). Mediciones de Estrés Laboral En Docentes de Un Colegio Público Regional Chileno. Measurements of Labor Stress in Teachers of a Regional Public High School., 29(1), 171-180.

Adil, M. S., \& Baig, M. (2018). Impact of Job Demands-Resources Model on Burnout and Employee's Well-Being: Evidence from the Pharmaceutical Organisations of Karachi. IIMB Management Review, 30(2), 119-133. https://doi.org/10.1016/j.iimb.2018.01.004

Bask, M., \& Salmela-Aro, K. (2013). Burned out to Drop out: Exploring the Relationship between School Burnout and School Dropout. European Journal of Psychology of Education, 28(2), 511-528. https://doi.org/10.1007/s10212-012-0126-5

Bottiani, J. H., Duran, C. A. K., Pas, E. T., \& Bradshaw, C. P. (2019). Teacher Stress and Burnout in Urban Middle Schools: Associations with Job Demands, Resources, and Effective Classroom Practices. Journal of School Psychology, 77, 36-51. https://doi. org/10.1016/j.jsp.2019.10.002

Buil, I., Martínez, E., \& Matute, J. (2019). Transformational Leadership and Employee Performance: The Role of Identification, Engagement and Proactive Personality. International Journal of Hospitality Management, 77, 64-75. https://doi.org/10.1016/j. ijhm.2018.06.014

Buunk, B. P., \& Schaufeli, W. B. (1993). Burnout: A Perspective from Social Comparison Theory. En Professional Burnout: Recent Developments in Theory and Research (pp. 53-69). Taylor \& Francis.

Caniëls, M. C. J., Semeijn, J. H., \& Renders, I. H. M. (2018). Mind the Mindset! The Interaction of Proactive Personality, Transformational Leadership and Growth Mindset for Engagement at Work. Career Development International, 23(1), 48-66. https:// doi.org/10.1108/CDI-11-2016-0194

Castillo-Gualda, R., Herrero, M., Rodríguez-Carvajal, R., Brackett, M. A., \& FernándezBerrocal, P. (2019). The Role of Emotional Regulation Ability, Personality, and Burnout among Spanish Teachers. International Journal of Stress Management, 26(2), 146.

Conte, J. M., Heffner, T. S., Roesch, S. C., \& Aasen, B. (2017). A Person-Centric Investigation of Personality Types, Job Performance, and Attrition. Personality and Individual Differences, 104, 554-559. https://doi.org/10.1016/j.paid.2016.09.004

Demir, S. (2018). The Relationship between Psychological Capital and Stress, Anxiety, Burnout, Job Satisfaction, and Job Involvement. Eurasian Journal of Educational Research, 18(75), 137-154.

Dhir, S., \& Shukla, A. (2019). Role of Organizational Image in Employee Engagement and Performance. Benchmarking: An International Journal, 26(3), 971-989. https://doi. org/10.1108/BIJ-04-2018-0094

Díaz, I. M. E., Tous-Pallarès, J., \& Vigil-Colet, A. (2015). Efecto Del Clima Psicosocial Del Grupo y de La Personalidad En El Síndrome de Quemado En El Trabajo En Docentes. Anales de Psicología/Annals of Psychology, 31(2), 651-657.

Dicke, T., Stebner, F., Linninger, C., Kunter, M., \& Leutner, D. (2018). A Longitudinal Study of Teachers' Occupational Well-Being: Applying the Job Demands-Resources Model. 
Journal of Occupational Health Psychology, 23(2), 262-277. https://doi.org/10.1037/ ocp0000070

Frajerman, A., Morvan, Y., Krebs, M.-O., Gorwood, P., \& Chaumette, B. (2019). Burnout in Medical Students before Residency: A Systematic Review and Meta-Analysis. European Psychiatry, 55, 36-42. https://doi.org/10.1016/j.eurpsy.2018.08.006

Fuller, M., Schadler, A., \& Cain, J. (2020). An Investigation of Prevalence and Predictors of Disengagement and Exhaustion in Pharmacy Students. American Journal of Pharmaceutical Education, 84(10), 1371-1377.

García-Carmona, M., Marín, M. D., \& Aguayo, R. (2019). Burnout Syndrome in Secondary School Teachers: A Systematic Review and Meta-Analysis. Social Psychology of Education, 22(1), 189-208. https://doi.org/10.1007/s11218-018-9471-9

Garcia-Jarillo, M., Francisco, C. D., \& Fayos, E. J. G. de los. (2016). El síndrome de burnout en deportistas: estudio piloto sobre la percepción del psicólogo deportivo a través del método Delphi. Cuadernos de Psicología del Deporte, 16(1), 243-250.

Hakanen, J. J., \& Bakker, A. B. (2017). Born and Bred to Burn out: A Life-Course View and Reflections on Job Burnout. Journal of occupational health psychology, 22(3), 354.

Huyghebaert, T., Gillet, N., Beltou, N., Tellier, F., \& Fouquereau, E. (2018). Effects of Workload on Teachers' Functioning: A Moderated Mediation Model Including Sleeping Problems and Overcommitment. Stress and Health, 34(5), 601-611. https://doi. org/10.1002/smi.2820

Iancu, A. E., Rusu, A., Măroiu, C., Păcurar, R., \& Maricuțoiu, L. P. (2018). The Effectiveness of Interventions Aimed at Reducing Teacher Burnout: A Meta-Analysis. Educational Psychology Review, 30(2), 373-396. https://doi.org/10.1007/s10648-017-9420-8

Jeung, D.-Y., Kim, C., \& Chang, S.-J. (2018). Emotional Labor and Burnout: A Review of the Literature. Yonsei medical journal, 59(2), 187.

Jovanovic, V., Hinic, D., Dzamonja-Ignjatovic, T., Stamatovic-Gajic, B., Gajic, T., \& Mihajlovic, G. (2020). Individual-Psychological Factors and Perception of Social Support in Burnout Syndrome. Vojnosanitetski pregled, 00, 4-4. https://doi.org/10.2298/ VSP190820004J

Khoeini, F., \& Attar, B. N. (2015). Personality Characteristics and Organizational Commitment in Iranian Employees. International Journal of Scientific Reports, 1(1), 61-64.

Kim, B., Lee, M., Kim, K., Choi, H., \& Lee, S. M. (2015). Longitudinal Analysis of Academic Burnout in Korean Middle School Students. Stress and Health, 31(4), 281-289.

Lee, Y. H. (2019). Emotional Labor, Teacher Burnout, and Turnover Intention in HighSchool Physical Education Teaching. European Physical Education Review, 25(1), 236-253. https://doi.org/10.1177/1356336X17719559

LePine, M. A., Zhang, Y., Crawford, E. R., \& Rich, B. L. (2015). Turning Their Pain to Gain: Charismatic Leader Influence on Follower Stress Appraisal and Job Performance. Academy of Management Journal, 59(3), 1036-1059. https://doi.org/10.5465/ amj.2013.0778 
Leupold, C. R., Lopina, E. C., \& Erickson, J. (2020). Examining the Effects of Core SelfEvaluations and Perceived Organizational Support on Academic Burnout Among Undergraduate Students. Psychological Reports, 123(4), 1260-1281. https://doi. org/10.1177/0033294119852767

Malinen, O.-P., \& Savolainen, H. (2016). The Effect of Perceived School Climate and Teacher Efficacy in Behavior Management on Job Satisfaction and Burnout: A Longitudinal Study. Teaching and Teacher Education, 60, 144-152. https://doi.org/10.1016/j. tate.2016.08.012

Mandrick, K., Peysakhovich, V., Rémy, F., Lepron, E., \& Causse, M. (2016). Neural and Psychophysiological Correlates of Human Performance under Stress and High Mental Workload. Biological Psychology, 121, 62-73. https://doi.org/10.1016/j.biopsycho.2016.10.002

Maslach, C., Schaufeli, W. B., \& Leiter, M. P. (2001). Job Burnout. Annual review of psychology, 52(1), 397-422.

Maticorena-Quevedo, J., Beas, R., Anduaga-Beramendi, A., \& Mayta-Tristán, P. (2016). Prevalencia Del Síndrome de Burnout En Médicos y Enfermeras Del Perú, ENSUSALUD 2014. Revista Peruana de Medicina Experimental y Salud Publica, 33(2), 241-247. https://doi.org/10.17843/rpmesp.2016.332.2170

Paredes, R. J. (2016). Síndrome de burnout y clima organizacional en obstetras del Servicio de Obstetricia del Hospital Nacional Daniel Alcides Carrión, Callao, 2015 [\{Tesis de maestr|'ia\}]. Universidad César Vallejo.

Paul III, D. P., Bakhamis, L., Smith, H., \& Coustasse, A. (2017). An American Epidemic: Burnout Syndrome in Hospital Nurses.

Pedersen, D. E., \& Minnotte, K. L. (2017). Workplace Climate and STEM Faculty Women's Job Burnout. Journal of Feminist Family Therapy, 29(1-2), 45-65. https:// doi.org/10.1080/08952833.2016.1230987

Perera, H. N., Granziera, H., \& McIlveen, P. (2018). Profiles of Teacher Personality and Relations with Teacher Self-Efficacy, Work Engagement, and Job Satisfaction. Personality and Individual Differences, 120, 171-178. https://doi.org/10.1016/j. paid.2017.08.034

Perestelo-Pérez, L. (2013). Standards on How to Develop and Report Systematic Reviews in Psychology and Health. International Journal of Clinical and Health Psychology, 13(1), 49-57. https://doi.org/10.1016/S1697-2600(13)70007-3

Rahim, A., \& Cosby, D. M. (2016). A Model of Workplace Incivility, Job Burnout, Turnover Intentions, and Job Performance. Journal of Management Development, 35(10), 1255-1265. https://doi.org/10.1108/JMD-09-2015-0138

Raja, U., Javed, Y., \& Abbas, M. (2018). A Time Lagged Study of Burnout as a Mediator in the Relationship between Workplace Bullying and WorkFamily Conflict. International journal of stress management, 25(4), 377. 
Rasool, S. F., Maqbool, R., Samma, M., Zhao, Y., \& Anjum, A. (2019). Positioning Depression as a Critical Factor in Creating a Toxic Workplace Environment for Diminishing Worker Productivity. Sustainability, 11(9), 2589. https://doi.org/10.3390/su11092589

Reyes, M. E. (2016). Relación Entre Habilidades Sociales y Desempeño Docente Desde La Percepción de Estudiantes Adultos de Universidad Privada En Lima, Perú. Revista Digital de Investigación en Docencia Universitaria, 10(2), 17-31. https://doi. org/10.19083/ridu.10.465

Robins, T. G., Roberts, R. M., \& Sarris, A. (2018). The Role of Student Burnout in Predicting Future Burnout: Exploring the Transition from University to the Workplace. Higher Education Research \& Development, 37(1), 115-130. https://doi.org/10.1080/ $\underline{07294360.2017 .1344827}$

Rodriguez-Díaz, M. A. (2010). Burnout: Un Fenómeno Creciente. Psicología desde el Caribe, 26, vii-x.

Rudolph, C. W., Katz, I. M., Lavigne, K. N., \& Zacher, H. (2017). Job Crafting: A MetaAnalysis of Relationships with Individual Differences, Job Characteristics, and Work Outcomes. Journal of Vocational Behavior, 102, 112-138. https://doi.org/10.1016/j. jvb.2017.05.008

Salanova, M., Martínez, I. M., \& Lorente, L. (2005). ¿Se Relacionan Los Obstáculos y Facilitadores Organizacionales Con El Burnout Docente?: Un Estudio Longitudinal. Revista de Psicología del Trabajo y de las Organizaciones, 21(1-2), 37-54.

Skaalvik, E. M., \& Skaalvik, S. (2018). Job Demands and Job Resources as Predictors of Teacher Motivation and Well-Being. Social Psychology of Education, 21(5), 12511275. https://doi.org/10.1007/s11218-018-9464-8

Sorkkila, M., Ryba, T. V., Selänne, H., \& Aunola, K. (2020). Development of School and Sport Burnout in Adolescent Student-Athletes: A Longitudinal Mixed-Methods Study. Journal of Research on Adolescence, 30(S1), 115-133. https://doi.org/10.1111/ jora. 12453

Swensen, S. J., \& Shanafelt, T. (2017). An Organizational Framework to Reduce Professional Burnout and Bring Back Joy in Practice. The Joint Commission Journal on Quality and Patient Safety, 43(6), 308-313. https://doi.org/10.1016/j.jcjq.2017.01.007

Teng, H.-Y., \& Chen, C.-Y. (2019). Proactive Personality and Job Crafting in the Tourism Industry: Does Job Resourcefulness Matter? Journal of Hospitality and Tourism Management, 41, 110-116. https://doi.org/10.1016/j.jhtm.2019.10.010

Treviño, E., Treviño, R., Segovia, S., Reyna, F., \& Vallejo, B. (2019). Burnout En Las Pymes: Una Aproximación Desde El Nivel Gerencial. AD-minister, 35, 137-163.

Upadyaya, K., Vartiainen, M., \& Salmela-Aro, K. (2016). From Job Demands and Resources to Work Engagement, Burnout, Life Satisfaction, Depressive Symptoms, and Occupational Health. Burnout Research, 3(4), 101-108. https://doi.org/10.1016/j. burn.2016.10.001

Van den Broeck, A., Elst, T. V., Baillien, E., Sercu, M., Schouteden, M., De Witte, H., \& Godderis, L. (2017). Job Demands, Job Resources, Burnout, Work Engagement, and 
Their Relationships: An Analysis Across Sectors. Journal of Occupational and Environmental Medicine, 59(4),369-376. https://doi.org/10.1097/JOM.0000000000000964

Vîrgă, D., Baciu, E.-L., Lazăr, T.-A., \& Lupșa, D. (2020). Psychological Capital Protects Social Workers from Burnout and Secondary Traumatic Stress. Sustainability, 12(6), 2246. https://doi.org/10.3390/su12062246

WHO. (2019). Burn-out an "Occupational Phenomenon": International Classification of Diseases. In Burn-out an "occupational phenomenon": International Classification of Diseases. https://www.who.int/news/item/28-05-2019-burn-out-an-occupationalphenomenon-international-classification-of-diseases.

Wilkerson, K., \& Bellini, J. (2006). Intrapersonal and Organizational Factors Associated with Burnout among School Counselors. Journal of Counseling \& Development, 84(4), 440-450.

Yin, H., Huang, S., \& Lv, L. (2018). A Multilevel Analysis of Job Characteristics, Emotion Regulation, and Teacher Well-Being: A Job Demands-Resources Model. Frontiers in Psychology, 9. https://doi.org/10.3389/fpsyg.2018.02395

Zarei, S., \& Dabaghi, P. (2019). Prevalence of Job Burnout Syndrome and Its Influential Factors among Military Personnel. Journal of Research and Health, 9(1), 45-52. https://doi.org/10.29252/jrh.9.1.45

Zysberg, L., Orenshtein, C., Gimmon, E., \& Robinson, R. (2017). Emotional Intelligence, Personality, Stress, and Burnout among Educators. International Journal of Stress Management, 24(Suppl 1), 122-136. https://doi.org/10.1037/str0000028

*Documentos sistematizados 